\title{
Envisioning Tangibles and Display-rich Interfaces for Co-located and Distributed Genomics Collaborations
}

\author{
Miriam K. Konkel \\ mkonkel@clemson.edu \\ Clemson University \\ Clemson, SC, USA \\ Orit Shaer \\ oshaer@wellesley.edu \\ Wellesley College \\ Wellesley, MA, USA
}

\author{
Brygg Ullmer \\ bullmer@clemson.edu \\ Clemson University \\ Clemson, SC, USA
Ali Mazalek
Ryerson University
Toronto, Canada \\ mazalek@ryerson.ca
}

\begin{abstract}
We explore how pervasive displays could offer unique opportunities for enhancing discovery and learning with "big data". In 2012-2014, our collaboration across three universities undertook a series of design exercises investigating approaches for collaborative, interactive, tangibles, and multitouch-engaged visualizations of genomic and related scientific datasets. These exercises led to several envisionments of tangible interfaces that employ active tokens and interactive surfaces to facilitate colocated and distributed engagement with large datasets. We describe some of the motivation and background for these envisioned interfaces; consider key aspects linking and distinguishing the designs; and relate these to the present and near-future state of the art for tangible and multitouch engagement with pervasive displays toward collaborative science.
\end{abstract}

\section{CCS CONCEPTS}

- Human-centered computing $\rightarrow$ User interface design; Interface design prototyping.

\section{KEYWORDS}

tangible genomics, collaborative genomic interfaces, tangible interfaces, pervasive collaborative scientific displays

\section{ACM Reference Format:}

Miriam K. Konkel, Brygg Ullmer, Orit Shaer, and Ali Mazalek. 2019. Envisioning Tangibles and Display-rich Interfaces for Co-located and Distributed Genomics Collaborations. In Proceedings of the 8th ACM International Symposium on Pervasive Displays (PerDis '19), June 12-14, 2019, Palermo, Italy. ACM, New York, NY, USA, 8 pages. https://doi.org/10.1145/3321335.3324953

\section{INTRODUCTION}

Both the first (2012) and latest (2019) "Pervasive Displays" venues frame pervasive displays in terms of a "new communication medium

Permission to make digital or hard copies of all or part of this work for personal or classroom use is granted without fee provided that copies are not made or distributed for profit or commercial advantage and that copies bear this notice and the full citation on the first page. Copyrights for components of this work owned by others than the author(s) must be honored. Abstracting with credit is permitted. To copy otherwise, or republish, to post on servers or to redistribute to lists, requires prior specific permission and/or a fee. Request permissions from permissions@acm.org.

PerDis '19, fune 12-14, 2019, Palermo, Italy

(c) 2019 Copyright held by the owner/author(s). Publication rights licensed to ACM. ACM ISBN 978-1-4503-6751-6/19/06 . .\$15.00

https://doi.org/10.1145/3321335.3324953 for public and semi-public spaces." While altogether a less common thread in this forum, the pursuit of science also is centrally concerned with communication in public and semi-public spaces. For example, Francis Crick (co-receiving a Nobel Prize for his contributions to the first characterization of DNA) asserted "communications is the essence of science" [21]. The context of DNA's characterization was not entirely unproblematic; it was partly made regarding Maurice Wilkins' sharing of the transformative "Photo 51" by Rosalind Franklin's doctoral student without Franklin's approval or knowledge [3]. But it does speak to the frequently collaborative nature of modern science. Illustrative examples include the 5,154 authors on a Higgs Boson paper [4]; more than 1,000 authors on the paper reporting the LIGO consortium's Nobel-winning observations of gravitational waves [5]; and (for one of the authors) participation among more than 700 co-authors on two high-impact human genomics papers $[18,50]$ (with more than 5,000 citations each).

In our experience, these large collaborative projects incorporate several facets and phases of "communication... in public and semi-public spaces." Loosely framed in terms of "when what is communicated with whom," early stages of scientific research can be seen as spanning a spectrum between private and semi-public. Even in the context of large consortium projects, students generally would share results with advisors and within their research group prior to sharing with wider audiences. An academic research lab could well be considered a semi-public space. Similarly, a broader research consortium (common for large science projects) that collaborates toward shared scientific ends also can be regarded as semi-public space.

Such work often is constrained by at least two forms of "embargoes," as one "red line" distinguishing "public" from "semipublic." In genomics contexts, the Bermuda [47], Ft. Lauderdale [2], and Toronto [13] agreements all govern conventions by which data, which is commonly made publicly available as it is generated, can be commonly engaged but not published in the interim (sometimes many years) without consent by the consortium before the first major "marker" publication is realized $[40,55]$. Press embargoes are also factors, but typically for much shorter periods [64].

Especially prior to pre-publication data embargoes, it is common for hundreds of researchers spanning dozens of institutions to vigorously collaborate for years. Tools to facilitate scientific dialogue, going beyond emailed slides before voice conference calls, 


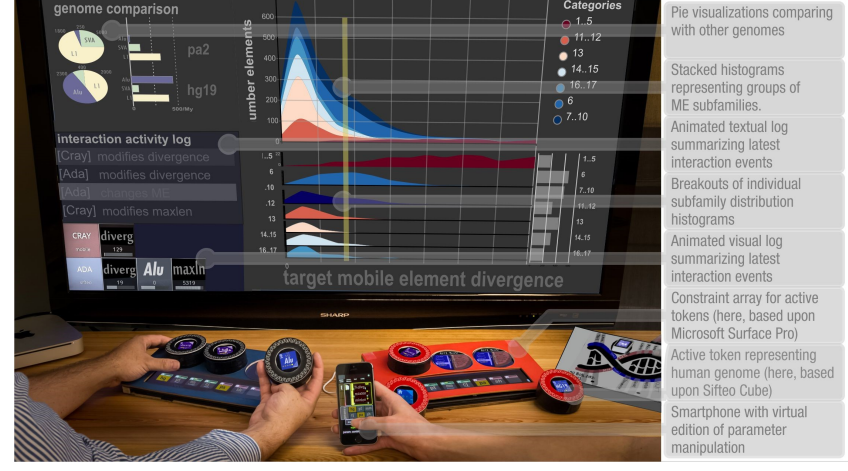

Figure 1: Envisionment of "Tabula” tangible genomics workbench (2014), tablet-based edition. Extracts from our existing genomics code $\&$ animated visual log prototypes integrated within a vertical screen, three tablets, six Sifteobased tokens, and a smartphone. (Sifteo cubes introduced in Section 4.) These physical tokens \& constraints leverage tangibility by supporting collaborative parameter token manipulation while eyes are oriented toward the shared screen or collaborators. Stacks of tokens were anticipated, per $[6,11$, 26, 71]. Bottom left: the Alu element is logged as impacted in an interaction event upon removal of the token.

hold potential for high scientific impact. Collaborating institutions commonly bring different disciplinary expertise, sometimes from different disciplines, increasing communication obstacles already posed by distance. This heterogeneity is often replicated in smaller form within individual research groups, both across seniority and disciplinary focus (e.g., computational methods vs. basic natural science).

Once work is published, a new ecosystem of "semi-public and public spaces" can be seen to exist. Some of these are in the context of formal education, be it postgraduate, undergraduate, or K-12. Others engage "broader impacts" outreach efforts, be they through museums, non-classroom K-12 activities, or others. Both for larger and more moderate government-funded scientific efforts (e.g., as with LIGO), these are often either encouraged or mandated as a condition of funding, toward eliciting greater engagement with students and the general public. Our genomics interests in particular hold criticality beyond basic "scientific literacy," to a more fundamental literacy that will reshape our medical care, and may even impact people's ability to hold employment or make prenatal or even preconception decisions $[10,62]$. Thus, this is of profound relevance to all humans. Here, too, "new communication medium[s] for public and semi-public spaces" hold special potential for impact.

To connect these discussions to the pervasive display domain, one of our envisioned, partially prototyped interfaces is illustrated in Figure 1. From the pervasive display vantage, we note the relative density and (in some respects) heterogeneity of interactive displays depicted within. Figure 1 represents (at least) one large, three medium, and eight small interactive displays manipulated by several individuals within a relatively small (several square meter) area. Similar technological resources might potentially span a much larger extent both in one physical site; and potentially bridge synchronous or asynchronous interactivity with interfaces at other physical sites (whether pairwise, tens, or even many thousands).

\section{BACKGROUND AND RELATED WORK}

One major challenge in computational genomics relates to the scale of datasets. Many genomic research efforts involve the study of multiple genomes. Today this may involve a thousand or more genomes in parallel, each containing billions of DNA base pairs. Soon, such scenarios may involve millions of genomes. There is a need for new computational tools for analysis and that facilitate meaningful interactive engagement with these vast datasets. Present interaction tools for computational genomics rarely venture beyond traditional graphical interaction techniques, thus missing the latent potential of alternate interaction paradigms.

Tangible and embodied interfaces (TEI) offer unique opportunities for enhancing the practice of computational genomics [13]. However, TEI research has not yet addressed scale and complexity of this magnitude. Understanding how to support more complex computations and flexible/scalable mappings between input and output has been recognized as an important challenge area to move the field forward [30].

Many arguments supporting tangible interfaces have been made, including cognitive, pedagogic, aesthetic, kinesthetic, and cultural [35, 49, 61, 69]. In parallel, important limitations for tangible interfaces remain. Challenges include the development of interfaces that go beyond one-to-one mapping, and provide means for searching, comparing, and sharing big data. Which representations are appropriate for large volumes of abstract data? What interaction techniques could facilitate fruitful exploration of big data? How can we effectively combine representations and manipulations to potentially reduce the mental workload associated with handling big data? Furthermore, as communication is frequently key to the success of genomic investigations, how can we best manage work across multiple co-located users, given the complex workflow and broad temporal range of interactions (from seconds to years)?

Direct touch has become a standard input method for tangible and multitouch interfaces. Yet, in data-intensive applications, representations are typically small [19]; here, finger size and occlusion make direct interaction difficult $[19,72,73]$. Also, in data-intensive applications, WIMP-style control elements provided by various multitouch toolkits, such as scrollbars, sliders, checkboxes, and text fields, may often be either too small for effective and accurate touch interaction, or consume relatively limited screen real estate [14, 19]. Several studies have considered novel multitouch interaction techniques for data-driven applications [14, 19, 33, 73]. While providing advantage over touch interaction with WIMP-style controls, multitouch gestures often suffer from low discoverability and lack of persistence [19]. We considered an alternative approach: exploring large data sets on multitouch and tangible surfaces using tangible interaction with active tokens, complemented by multitouch and gestural interaction.

Active tokens are programmable physical objects with integrated display, sensing, or actuation technologies [48, 71, 77]. Thus, they can be reconfigured over time, allowing users to dynamically modify their associations with datasets or controls. Users can thereby choose and evolve appropriate tools over successive stages of (e.g.) 
scientific workflows. Active tokens can also be arranged in various spatial configurations, utilizing physical syntax to represent complex information workflows. The majority of tangible interfaces to date have, from a human sensory perspective, employed passive physical tokens. While these artifacts have often been embedded with various forms of tags and sensors, mediation has typically been via active surfaces illuminated internally, from beneath, or above. While they can support perceptual coupling of bits and atoms [67] or "coincidence of input and output space" [34] while on such surfaces, passive tokens are often perceptually divorced from their digital associations when in hand (above a surface) and in reserve (on or outside of surface bezels). Especially in big data domains, the number of available tangibles is likely to be dwarfed by their potential range of digital bindings (or "cyberphysical associations"). Active tokens hold the potential to address these and other important limitations.

Here, we focus on a subclass of active tokens that can be manipulated both within mechanical constraints, and using gestures independently from such constraints. These kinds of active tokens enable the expansion of tangible interaction with multitouch and tangible surfaces beyond interaction on the surface into less explored areas such as tangible interaction on bezel, in air, hovering above, or in front the surface. Expanding interaction with active tokens beyond the surface could free much needed real estate for visual data representations, among other potential benefits.

\section{COMPUTATIONAL GENOMICS \& BIG DATA}

While TEIs designed for large data sets can apply to many different areas, we have chosen computational genomics as a target domain for our research for several reasons. Advances in genomic technologies have transformed biological inquiry, and have begun to revolutionize medical practice to offer muchimproved healthcare $[16,17]$. For example, cancer treatment is now often individually tailored toward the genetics of the cancer, highlighting the potential of precision medicine and providing a glimpse into the future of medical treatment. Also, genomic and biological technologies are positioned to address some of the most pressing problems of our times, including food and clean water shortages, as well as increased demand for alternative energy sources [24]. Further, the field of genomic technologies has opened new interfaces between biology and computer science, fueling fields such as bioinformatics that enable biological questions to be tackled computationally [17].

Resonant with broader evolutions in science $[58,75]$, the study of genomes now engages theory, experimentation, and computation on equal footing. The combination of advanced genomic technologies (e.g. high-throughput DNA sequencing) and powerful computational tools has facilitated biological investigations in previously impossible manners and scales [46]. No longer limited to small-scale analyses (e.g., of a few genes or specific genomic regions), researchers now often conduct largescale experiments where information from multiple genomes is measured, recorded, analyzed, and stored. The bottlenecks and challenges along the path to transforming the "big data" generated by these experiments into biological insights have shifted from data generation to data analysis $[17,62]$. These have highlighted the need for new computational tools that facilitate effective, meaningful, collaborative analyses

\subsection{TEI systems for scientific understanding}

A number of systems illustrate possibilities for supporting scientific discovery and higher education with TEI. Brooks et al. [56] developed the first haptic display for scientific visualization. Gillet et al. [24] presented a tangible user interface for molecular biology that used augmented reality technology to view 3D molecular models. Schkolne et al. [59] developed an immersive tangible interface for the design of DNA molecules. Grote et al. developed a tangible user interface for bio-design that supports a scientific workflow that requires the exploration of large datasets through the construction of complex queries [26]. While these systems highlight potential benefits of TEI for scientists, they mostly focus on the representation of objects with inherent physical structure. We are interested in a broader use case, where abstract information (for which no intrinsic spatial representation typically exists) is represented and manipulated.

Several projects investigate augmented capture and situated access to biological data. Labscape [9] is a smart environment for cell biology labs. ButterflyNet [76] is a mobile capture and access system for field biologists. Mackay et al. and Tabard et al. [45, 65] explore the integration of biologists' notebooks with physical + digital information sources. While these systems demonstrate the feasibility of augmenting experimental workflows, our focus in these efforts has been upon transforming data into insights.

Other systems have been developed to facilitate collaboration among co-located teams of scientists across large displays and multitouch tables. WeSpace [74] integrates a large data wall with a multitouch table and personal laptops. TeamTag [54] allows biodiversity researchers to collaboratively search, label, and browse digital photos. Isenberg et al. studied collaborative visual analytics [32]. eLabBench [65] investigated tabletop interfaces as interactive wet lab benches. Kuznetsov et al. explored the development of artifacts for supporting DIYbio [42]. Other related resources include coordination policies and guidelines for colocated groupware [49, 50] and evaluation methodologies for collaborative environments $[31,38,63]$.

TEI systems have also demonstrated potential to support science education. Those most relevant to genomics include Augmented Chemistry [20], a tangible user interface for chemistry education; Involv [29], a tabletop interface for exploring the Encyclopedia of Life that shares our challenge of creating effective interaction techniques for large data spaces; and PhyloGenie [60], a tabletop interface for collaborative learning of phylogeny through guided activity. In contrast to these works, we are interested in the development of interfaces that empower both expert and novice researchers to conduct open-ended hands-on inquiry.

\section{TANGIBLE GENOMICS ENVIRONMENTS}

With this background, we turn to several envisionments of prospective tangible genomics interaction environments.

Both of our envisionments have been framed in the context of a several square meter workspace. Each could easily (and perhaps 


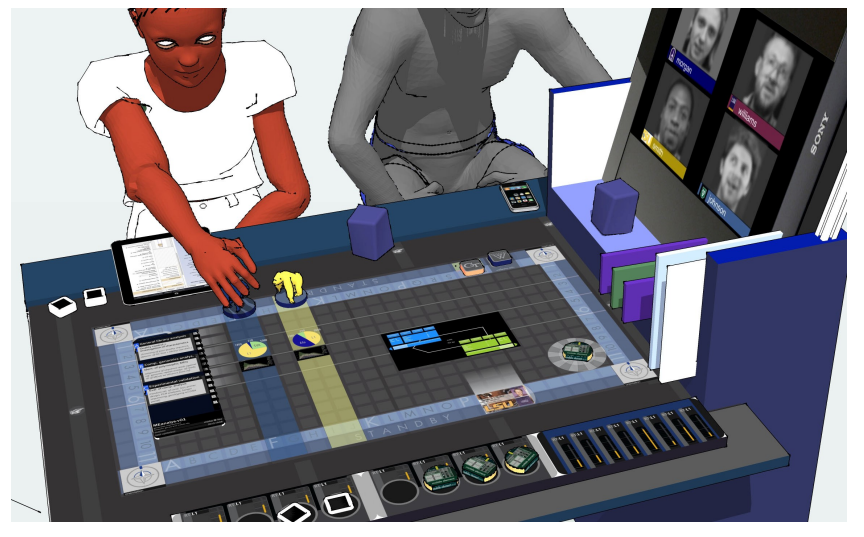

Figure 2: "Tabula" tangible genomics workbench (2012).

preferably) be room-spanning. As each was anticipated to be installed within at least three different university contexts, we tended toward self-contained prototypes. Both systems reflect our interests in integrating mass-market commodity devices, including several technologies specific to the period. For example, our research programs had each engaged Sifteo Cubes [44, 51-53, 57]. These "cubes" (in actuality, 1.5 inch x 1.5 inch x 0.75 inch) contain touch screens and are motion- and proximity-aware. While designed as gaming devices, Sifteo went open source and allows the development of non-game content. Sifteo Cubes use gestures - including tilt, shake, neighbor, press, wiggle, slide, flip, and stack - as modes of interactions.

While a compelling platform for continuing work - in our case, also incorporating special capabilities coordinated with the manufacturer - the Sifteo technology was acquired and discontinued. By the time of Figure 1's creation, we expressed our intent to consider (e.g.) smart watches and small form factor smartphones as alternatives. Similarly, Figure 2 centers around the form factor and functional properties of the Microsoft PixelSense/Samsung SUR40 device. SUR40, too, was short-lived; Figure 1 excised its inclusion.

The name of our envisioned system, Tabula, was used for roughly 1,000 years as a term for medieval European "counting tables" - a calculating approach somewhat reminiscent of the abacus, and a predecessor to computational spreadsheets. The information visualization spreadsheet concept [62] also seemed congruent to both multitouch and tangibles use.

We sought to provide paths for employing tangibles to represent the "key objects of interest" [61,68]. Several specific planned variations included:

- Some tangibles are used to represent data; others, tools.

- Some proposed tangibles are passive; others, active (e.g., incorporating sensing and displays).

- Some tangibles are physically representational (e.g., Figure 3a; representing different kinds of primates); others, visually representational (Figure $3 \mathrm{~b}$, representing different campuses); others, physically and visually abstract.

- Some interactive elements are physical, others virtual. E.g., Figure 3b illustrates both "hard" (physical) and "soft" (virtual) tokens representing different campuses.
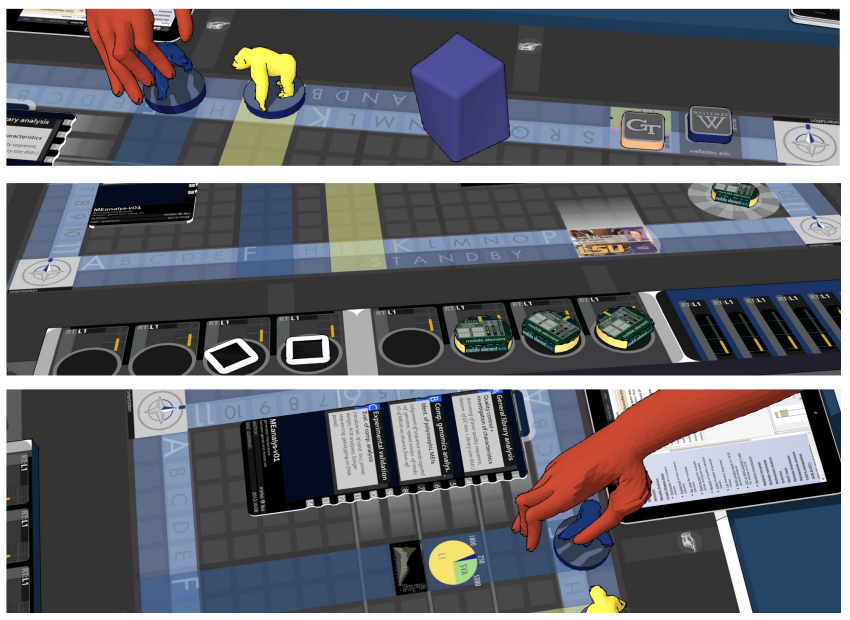

Figure 3: a) 2012 envisionment inset 1: genome, genome panel, place/team tangibles; $b$ ) inset 2 : active and passive parameter tokens: ID as container; parameter pie menus; c) inset 3: workflow tangibles; tablet with linked Galaxy workflow; visualizations

Rather than expecting all aspects of the interface to be physically embodied, we instead envisioned many system facets at different stages flowing between representation in physical and virtual forms. Thus, we sought to take advantage of digital malleability and proactivity evident in (e.g.) predictive web search, while also engaging the benefits of tangible interaction.

\subsection{Prospective Elements}

Both in research and teaching labs and in the classroom, we envisioned Tabula engaging $\sim 6-12$ active tokens, and one or several interactive surfaces. Each active token could take on various functional bindings. Several prospects are summarized in Table 1. Active tokens were envisioned to combine with constraint cartouches [66] in several ways. First, they could be bound to different associations manually. E.g., using a two-handed interaction, a user might touch a binding on a tablet or tabletop with one hand, and depress a target token with the other hand. Second, active tokens could be manipulated within constraints to operate upon token bindings. In Table 1's examples, rotating token \#1 could select between several available primate genomes (marmoset, gibbon, etc.); rotating \#6, expressing a mobile element's full-length threshold (a process which typically requires iterative manipulation to parametrically select anywhere from a handful to hundreds of thousands of target elements). In addition to passive haptic feedback from turning the token, we envision providing active visual feedback on the token itself, on the backing interactive surface, and on a proximal vertical display. These are intended to support evolving views by multiple collaborating users.

\subsection{Sample Interactions}

Figure 1 and 4 illustrate a prospective interaction comparing Platy1 mobile elements (a recently discovered mobile element specific to New World monkeys $[15,41])$ within the marmoset genome. 


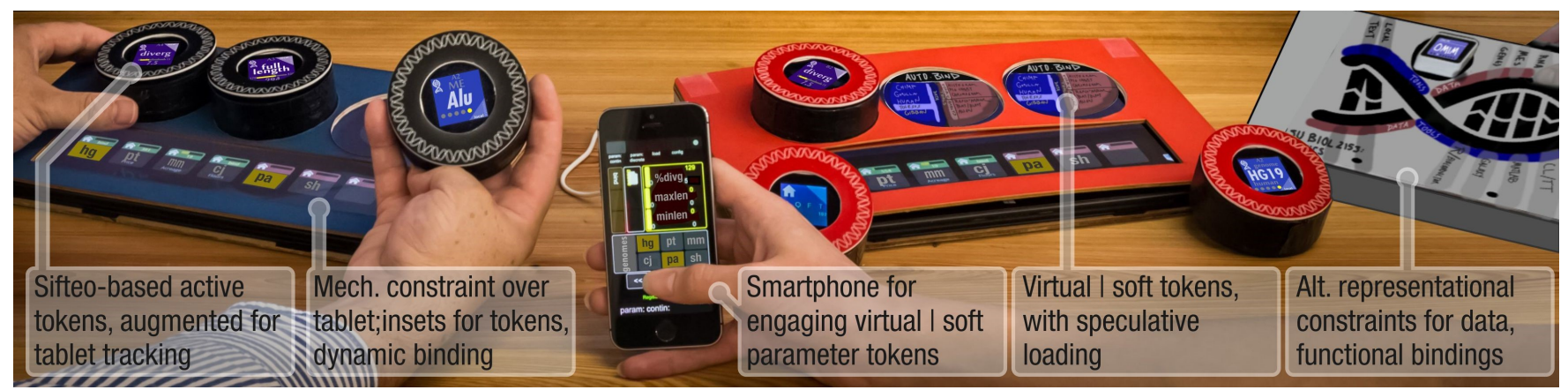

Figure 4: 2014 envisionment inset: Envisionment of Tabula prototype (one edition; inset of Figure 1). Several active tokens are present within mechanically constraining wells within physical cartouche constraints fixtured upon tablets. Two wells are physically empty, but graphically auto-filled with predictive data and tool suggestions, including a default selection. Two of the tablets (relabeled versions of working prototypes) incorporate relatively generic token constraints. A third (envisioned on right) explores more representational finger constraints for relatively persistent data, function bindings.

Table 1: Examples bindings for Tabula active tokens. Background colors indicate two classes - data and tools; shading indicates subclasses. Some tokens might be bound to a single association; others, to small or large aggregates.

\begin{tabular}{ccll} 
tok \# & class & binding & exampl. contents \\
\hline 1 & data & genome & $\mathrm{n}$ primates \\
2 & data & mobile element (ME)type & $\mathrm{n}$ ME classes \\
3 & data & mobile element type & $\mathrm{n}$ kothi variants \\
4 & data & candidate element instance & 47 elements \\
5 & tools & analytics tools & RepeatMasker, etc. \\
6 & tools & param & length threshold
\end{tabular}

Rotating physical tokens, or engaging finger-constraining interactions in the empty token wells, were envisioned as allowing the addition or changing of genomes, targeting of mobile elements, and assignment of full-length thresholds. Additional visualizations, primer design, computational analyses, and other actions would be physically or graphically invoked and parametrically controlled through similar interactions. Active tokens could be lifted from the workspace and held, placed, or exchanged with other users to support varying styles of epistemic cognition [39]. Tokens might be virtually or physically brought to (e.g.) a laptop for manipulation in a conventional spreadsheet, or to a wall-scale display for presentation use.

\section{DISCUSSION AND FUTURE WORK}

In some respects, the 2014 variation of Figure 1 expresses a subset of the representational forms and proposed functions of Figure 2's 2012 version. Where the 2012 version was targeted toward distributed collaboration, the 2014 version was focused on co-located interaction. Where the 2012 version incorporated both physically representational and abstracted tangibles, the 2014 version was populated primarily with abstracted tangibles. And where the 2012 version anticipated ambitious use of the tabletop display, this was removed from the 2014 version.
That said, to our knowledge, both the 2012 and 2014 interface envisionments illustrated and aspired to a more ambitious set of digital functionality (at minimum, within the context of computational science) and diversity of integrated displays than any to-date tangibles interface of which we are aware. One interpretation is a platform/content tension alluded by Ansoff [7], articulated by Merrill, and elaborated within [69]. Implementing the hardware alone of Figure 1 or 2 is an ambitious proposition, as would be the software alone. Especially with the resources of academic contexts, a direct ad-hoc de novo creation of the full hardware and software ecosystem is likely to be challenging and fraught. Our team realized this, and sought to position existing software environments like Galaxy $[23,25,27]$ - an open source environments for genomic analyses targeted toward non-programmers - and platforms like Sifteo and PixelSense [22, 52]. But we also noted a relatively wide functional and API gap between Galaxy and our needs. Also, the Sifteo and PixelSense platforms were already in rapid decline, and the Sifteo remains currently without a commercially available successor.

At the time, our team identified tangible reinterpretations of smart watches as one promising vector. Some of us have pursued this further [8], with some success. Some of us have also partially developed active tokens utilizing ePaper and NeoPixel rings, which could also offer a complementary platform. In all cases, the rapid turnover of hardware platforms (as with smart watches), and the resource demands of platform development, remain significant obstacles. The creation of interoperable virtual editions - both on 2D screens, and also in VR environments - remains one attractive path, if partly as a bootstrapping vector. At the same time, we anticipate a careful balancing act must be made. If, as in our passage from the 2012 to 2014 prototypes (or in another example, the evolution of the Urp tangible interface from initial to classroom-deployed form $[12,70])$, there is too much functional and representational dilution, the result may be insufficiently compelling to attract and sustain use and development.

In the present and near-future, we see several promising prospects toward catalyzing the creation of such functionalities. New mediation technologies such as LightCrafter (used for positional sensing by Zooids [43]), in combination with active tokens, could provide 
paths for tabletop mediation and sensing with newly compelling capabilities and economics. The combination of small, inexpensive ePaper modules and embedded computers (e.g., WiFi integrated Arduinos), combined with 3D printing, could change some of the platform dynamics underlying active tokens. The rapid growth and investments of VR, combined with compelling complementarities between VR and tangible interfaces [28], could drive the creation of software platforms that could accelerate creating such environments.

Continuing evolutions in the smartwatch space - e.g., decrease in the cost of high-function legacy devices, perhaps as inbuilt batteries of Apple watches fail, or as Android variants gain functionality and traction - could reshape the active token landscape. Decreasing costs and new technologies in the sensate large-screen landscape is another driver. The accelerating trajectories of both personal genomics and genomics within academic research - and corresponding demands for and software platforms enabling new interactive modes for engaging genomics - would also be a powerful complementary driver. In a final variation, where low-level protocols like TUIO $[1,36,37]$ achieved substantial impact and uptake, higher-level sister APIs - perhaps initially in the context of games, music, and other mass drivers - could substantially ease system development for ambitious tangible pervasive displays.

\section{ACKNOWLEDGMENTS}

We are appreciative of NSF grants CNS-1828611, CNS-1126739, IIS1149530, and IIS-1320350; the NSERC Discovery Grant and Canada Research Chairs programs, and the Canada Foundation for Innovation (CFI) and Ontario Ministry of Research and Innovation (MRI) Innovation Fund for partial support of this work. We also thank David Merrill, Liam Staskawicz, Consuelo Valdes, Casey Grote, André Wiggins, and Michael Lynn for supporting this work.

\section{REFERENCES}

[1] [n. d.]. Software implementierung TUIO. http://www.tuio.org/?software

[2] 2003. Sharing data from large-scale biological research projects: a system of tripartite responsibility. http://www.wellcome.-ac.uk/stellent/groups/corporatesite/ @policy_communications/documents/web_document/wtd003207.pdf.

[3] 2013. Due credit. Nature 496, 7445 (2013), 270. https://doi.org/10.1038/496270a

[4] G. Aad, B. Abbott, J. Abdallah, and et al. 2015. Combined Measurement of the Higgs Boson Mass in $p p$ Collisions at $\sqrt{s}=7$ and $8 \mathrm{TeV}$ with the ATLAS and CMS Experiments. Phys. Rev. Lett. 114 (May 2015), 191803. Issue 19. https: //doi.org/10.1103/PhysRevLett.114.191803

[5] B. P. Abbott, R. Abbott, T. D. Abbott, and et al. 2016. Observation of Gravitational Waves from a Binary Black Hole Merger. Phys. Rev. Lett. 116 (Feb 2016), 061102 Issue 6. https://doi.org/10.1103/PhysRevLett.116.061102

[6] Anand Agarawala and Ravin Balakrishnan. 2006. Keepin' It Real: Pushing the Desktop Metaphor with Physics, Piles and the Pen. In Proceedings of the SIGCHI Conference on Human Factors in Computing Systems (CHI '06). ACM, New York, NY, USA, 1283-1292. https://doi.org/10.1145/1124772.1124965

[7] H Igor Ansoff et al. 1957. Strategies for diversification. Harvard business review 35, 5 (1957), 113-124.

[8] Ahmed Sabbir Arif, Roozbeh Manshaei, Sean Delong, Brien East, Matthew Kyan, and Ali Mazalek. 2016. Sparse Tangibles: Collaborative exploration of gene networks using active tangibles and interactive tabletops. In Proceedings of the TEI'16: Tenth International Conference on Tangible, Embedded, and Embodied Interaction. ACM, 287-295.

[9] L. Arnstein, Chia-Yang Hung, R. Franza, Qing Hong Zhou, G. Borriello, S. Consolvo, and Jing Su. 2002. Labscape: a smart environment for the cell biology laboratory. IEEE Pervasive Computing 1, 3 (July 2002), 13-21. https: //doi.org/10.1109/MPRV.2002.1037717

[10] Pierre Baldi, Roberta Baronio, Emiliano De Cristofaro, Paolo Gasti, and Gene Tsudik. 2011. Countering GATTACA: Efficient and Secure Testing of Fullysequenced Human Genomes. In Proceedings of the 18th ACM Conference on Computer and Communications Security (CCS '11). ACM, New York, NY, USA, 691-702. https://doi.org/10.1145/2046707.2046785

[11] Tom Bartindale and Chris Harrison. 2009. Stacks on the Surface: Resolving Physical Order Using Fiducial Markers with Structured Transparency. In Proceedings of the ACM International Conference on Interactive Tabletops and Surfaces (ITS '09). ACM, New York, NY, USA, 57-60. https://doi.org/10.1145/1731903.1731916

[12] Eran Ben-Joseph, Hiroshi Ishii, John Underkoffler, Ben Piper, and Luke Yeung. 2001. Urban simulation and the luminous planning table: Bridging the gap between the digital and the tangible. Fournal of planning Education and Research 21, 2 (2001), 196-203.

[13] Ewan Birney, Thomas J Hudson, Eric D Green, Chris Gunter, Sean Eddy, Jane Rogers, Jennifer R Harris, S Dusko Ehrlich, Rolf Apweiler, Christopher P Austin, et al. 2009. Prepublication data sharing. Nature 461, 7261 (2009), 168-170.

[14] F. Block, M. S. Horn, B. C. Phillips, J. Diamond, E. M. Evans, and C. Shen. 2012. The DeepTree Exhibit: Visualizing the Tree of Life to Facilitate Informal Learning. IEEE Transactions on Visualization and Computer Graphics 18, 12 (Dec 2012), 2789-2798. https://doi.org/10.1109/TVCG.2012.272

[15] Lucia Carbone, R Alan Harris, Alan R Mootnick, Aleksandar Milosavljevic, David IK Martin, Mariano Rocchi, Oronzo Capozzi, Nicoletta Archidiacono, Miriam K Konkel, Jerilyn A Walker, et al. 2012. Centromere remodeling in Hoolock leuconedys (Hylobatidae) by a new transposable element unique to the gibbons. Genome biology and evolution 4, 7 (2012), 648-658.

[16] Rui Chen, George I. Mias, Jennifer Li-Pook-Than, and et al. 2012. Personal Omics Profiling Reveals Dynamic Molecular and Medical Phenotypes. Cell 148, 6 (2012), 1293 - 1307. https://doi.org/10.1016/j.cell.2012.02.009

[17] Lynda Chin, William C. Hahn, Gad Getz, and Matthew Meyerson. 2011. Making sense of cancer genomic data. Genes \& Development 25, 6 (2011), 534-555. https://doi.org/10.1101/gad.2017311 arXiv:http://genesdev.cshlp.org/content/25/6/534.full.pdf+html

[18] Genomes Project Consortium. 2010. A map of human genome variation from population-scale sequencing. Nature 467, 7319 (2010), 1061-1073.

[19] Steven M. Drucker, Danyel Fisher, Ramik Sadana, Jessica Herron, and m.c. schraefel. 2013. TouchViz: A Case Study Comparing Two Interfaces for Data Analytics on Tablets. In Proceedings of the SIGCHI Conference on Human Factors in Computing Systems (CHI '13). ACM, New York, NY, USA, 2301-2310. https://doi.org/10.1145/2470654.2481318

[20] Morten Fjeld, Jonas Fredriksson, Martin Ejdestig, Florin Duca, Kristina Bötschi, Benedikt Voegtli, and Patrick Juchli. 2007. Tangible User Interface for Chemistry Education: Comparative Evaluation and Re-design. In Proceedings of the SIGCHI Conference on Human Factors in Computing Systems (CHI'07). ACM, New York, NY, USA, 805-808. https://doi.org/10.1145/1240624.1240745

[21] William D Garvey. 2014. Communication: the essence of science: facilitating information exchange among librarians, scientists, engineers and students. Elsevier.

[22] Luc Geurts, Vero Vanden Abeele, Kevin Van Keer, and Ruben Isenborghs. 2014. Playfully Learning Visual Perspective Taking Skills with Sifteo Cubes. In Proceedings of the First ACM SIGCHI Annual Symposium on Computer-human Interaction in Play (CHI PLAY '14). ACM, New York, NY, USA, 107-113. https: //doi.org/10.1145/2658537.2658706

[23] Belinda Giardine, Cathy Riemer, Ross C Hardison, Richard Burhans, Laura Elnitski, Prachi Shah, Yi Zhang, Daniel Blankenberg, Istvan Albert, James Taylor, et al. 2005. Galaxy: a platform for interactive large-scale genome analysis. Genome research 15, 10 (2005), 1451-1455.

[24] A. Gillet, M. Sanner, D. Stoffler, and A. Olson. 2005. Tangible augmented interfaces for structural molecular biology. IEEE Computer Graphics and Applications 25, 2 (March 2005), 13-17. https://doi.org/10.1109/MCG.2005.47

[25] Jeremy Goecks, Anton Nekrutenko, and James Taylor. 2010. Galaxy: a comprehensive approach for supporting accessible, reproducible, and transparent computational research in the life sciences. Genome biology 11, 8 (2010), R86.

[26] Casey Grote, Evan Segreto, Johanna Okerlund, Robert Kincaid, and Orit Shaer. 2015. Eugenie: Multi-Touch and Tangible Interaction for Bio-Design. In Proceedings of the Ninth International Conference on Tangible, Embedded, and Embodied Interaction (TEI '15). ACM, New York, NY, USA, 217-224. https://doi.org/10.1145/ 2677199.2680605

[27] BjÃ̂ürn A. GrÃijning, Eric Rasche, Boris Rebolledo-Jaramillo, Carl Eberhard, Torsten Houwaart, John Chilton, Nate Coraor, Rolf Backofen, James Taylor, and Anton Nekrutenko. 2017. Jupyter and Galaxy: Easing entry barriers into complex data analyses for biomedical researchers. PLOS Computational Biology 13, 5 (05 2017), 1-10. https://doi.org/10.1371/journal.pcbi.1005425

[28] Daniel Harley, Aneesh P Tarun, Daniel Germinario, and Ali Mazalek. 2017. Tangible vr: Diegetic tangible objects for virtual reality narratives. In Proceedings of the 2017 Conference on Designing Interactive Systems. ACM, 1253-1263.

[29] M. S. Horn, M. Tobiasz, and C. Shen. 2009. Visualizing Biodiversity with Voronoi Treemaps. In 2009 Sixth International Symposium on Voronoi Diagrams. 265-270. https://doi.org/10.1109/ISVD.2009.22

[30] E. Hornecker, R. J. K. Jacob, C. Hummels, B. Ullmer, A. Schmidt, E. van den Hoven, and A. Mazalek. 2008. TEI goes on: tangible and embedded interaction. IEEE Pervasive Computing 7, 2 (2008), 91-95. https://strathprints.strath.ac.uk/32703/ 
[31] Kori M Inkpen. [n. d.]. âĂIJJust BecauseâĂİ The Challenges of Evaluating Faceto-Face Collaboration. ([n. d.])

[32] P. Isenberg, D. Fisher, M. R. Morris, K. Inkpen, and M. Czerwinski. 2010. An exploratory study of co-located collaborative visual analytics around a tabletop display. In 2010 IEEE Symposium on Visual Analytics Science and Technology. 179-186. https://doi.org/10.1109/VAST.2010.5652880

[33] P. Isenberg, T. Isenberg, T. Hesselmann, B. Lee, U. von Zadow, and A. Tang. 2013 Data Visualization on Interactive Surfaces: A Research Agenda. IEEE Computer Graphics and Applications 33, 2 (March 2013), 16-24. https://doi.org/10.1109/ MCG.2013.24

[34] Hiroshi Ishii. 2008. Tangible Bits: Beyond Pixels. In Proceedings of the 2Nd International Conference on Tangible and Embedded Interaction (TEI '08). ACM, New York, NY, USA, xv-xxv. https://doi.org/10.1145/1347390.1347392

[35] Hiroshi Ishii and Brygg Ullmer. 1997. Tangible bits: towards seamless interfaces between people, bits and atoms. In Proceedings of the ACM SIGCHI Conference on Human factors in computing systems. ACM, 234-241.

[36] Martin Kaltenbrunner. 2009. reacTIVision and TUIO: a tangible tabletop toolkit. In Proceedings of the ACM international Conference on interactive Tabletops and Surfaces. ACM, 9-16.

[37] Martin Kaltenbrunner, Till Bovermann, Ross Bencina, Enrico Costanza, et al 2005. TUIO: A protocol for table-top tangible user interfaces. In Proc. of the The 6th International Workshop on Gesture in Human-Computer Interaction and Simulation. 1-5.

[38] David Kirsh. 2004. Methodologies for Evaluating Collaboration Behavior in Co-Located Environments. CSCW 2004 Workshop: Methodologies for Evaluating Collaboration in Co-Located Environments.

[39] David Kirsh and Paul Maglio. 1994. On distinguishing epistemic from pragmatic action. Cognitive science 18, 4 (1994), 513-549.

[40] D. Koboldt. 2013. Data Sharing, Embargo, and Big Science. http://massgenomics. org/2013/06/data-sharing-embargo.html.

[41] Miriam K Konkel, Brygg Ullmer, Erika L Arceneaux, Sreeja Sanampudi, Sarah A Brantley, Robert Hubley, Arian FA Smit, and Mark A Batzer. 2016. Discovery of a new repeat family in the Callithrix jacchus genome. Genome research 26, 5 (2016), 649-659.

[42] Stacey Kuznetsov, Alex S. Taylor, Tim Regan, Nicolas Villar, and Eric Paulos. 2012. At the Seams: DIYbio and Opportunities for HCI. In Proceedings of the Designing Interactive Systems Conference (DIS '12). ACM, New York, NY, USA 258-267. https://doi.org/10.1145/2317956.2317997

[43] Mathieu Le Goc, Lawrence H Kim, Ali Parsaei, Jean-Daniel Fekete, Pierre Dragicevic, and Sean Follmer. 2016. Zooids: Building blocks for swarm user interfaces. In Proceedings of the 29th Annual Symposium on User Interface Software and Technology. ACM, 97-109.

[44] S. Perry L. Staskawicz M. E. Scott, M. K. [n. d.]. Github: sifteo / thundercracker. https://github.com/sifteo/thundercracker.

[45] Wendy E. Mackay, Guillaume Pothier, Catherine Letondal, Kaare Bøegh, and Hans Erik Sørensen. 2002. The Missing Link: Augmenting Biology Laboratory Notebooks. In Proceedings of the 15th Annual ACM Symposium on User Interface Software and Technology (UIST '02). ACM, New York, NY, USA, 41-50. https: //doi.org/10.1145/571985.571992

[46] Elaine R. Mardis. 2008. The impact of next-generation sequencing technology on genetics. Trends in Genetics 24, 3 (2008), 133 - 141. https://doi.org/10.1016/j. tig.2007.12.007

[47] Eliot Marshall. 2001. Bermuda Rules: Community Spirit, With Teeth. Science 291, 5507 (2001), 1192-1192. https://doi.org/10.1126/science.291.5507.1192 arXiv:https://science.sciencemag.org/content

[48] Ali Mazalek, Orit Shaer, Brygg Ullmer, Miriam K Konkel, et al. 2014. Tangible meets gestural: Gesture based interaction with active tokens. In ACM CHI 2014 Workshop on Gesture-based Interaction Design, ACM CHI.

[49] Ali Mazalek and Elise Van Den Hoven. 2009. Framing tangible interaction frameworks. AI EDAM 23, 3 (2009), 225-235

[50] GA McVean. 2012. RM D (CC), Abecasis GR, Bentley DR, Chakravarti A, McVean GA, Altshuler DM, Durbin RM, Abecasis GR, Bentley DR, Chakravarti A, et al An integrated map of genetic variation from 1,092 human genomes. Nature 491 (2012), 56-65

[51] David Merrill, Jeevan Kalanithi, and Pattie Maes. 2007. Siftables: Towards Sensor Network User Interfaces. In Proceedings of the 1st International Conference on Tangible and Embedded Interaction (TEI '07). ACM, New York, NY, USA, 75-78. https://doi.org/10.1145/1226969.1226984

[52] David Merrill, Emily Sun, and Jeevan Kalanithi. 2012. Sifteo cubes. In CHI'12 Extended Abstracts on Human Factors in Computing Systems. ACM, 1015-1018.

[53] David Jeffrey Merrill. 2009. Interaction with embodied media. Ph.D. Dissertation.

[54] Meredith Ringel Morris, Andreas Paepcke, Terry Winograd, and Jeannie Stamberger. 2006. TeamTag: Exploring Centralized Versus Replicated Controls for Co-located Tabletop Groupware. In Proceedings of the SIGCHI Conference on Human Factors in Computing Systems (CHI '06). ACM, New York, NY, USA, 1273-1282. https://doi.org/10.1145/1124772.1124964

[55] Shreeya Nanda and Maria K Kowalczuk. 2014. Unpublished genomic data-how to share?
[56] M. Ouh-young, M. Pique, J. Hughes, N. Srinivasan, and F. P. Brooks. 1988. Using a manipulator for force display in molecular docking. In Proceedings. 1988 IEEE International Conference on Robotics and Automation. 1824-1829 vol.3. https: //doi.org/10.1109/ROBOT.1988.12330

[57] Clément Pillias, Raphaël Robert-Bouchard, and Guillaume Levieux. 2014. Designing Tangible Video Games: Lessons Learned from the Sifteo Cubes. In Proceedings of the 32Nd Annual ACM Conference on Human Factors in Computing Systems (CHI '14). ACM, New York, NY, USA, 3163-3166. https://doi.org/10.1145/2556288. 2556991

[58] Daniel A Reed, Ruzena Bajcsy, Manuel A Fernandez, Jose-Marie Griffiths, Randall D Mott, Jack Dongarra, Chris R Johnson, Alan S Inouye, William Miner, Martha K Matzke, et al. 2005. Computational science: ensuring America's competitiveness. Technical Report. PRESIDENT'S INFORMATION TECHNOLOGY ADVISORY COMMITTEE ARLINGTON VA.

[59] Steven Schkolne, Hiroshi Ishii, and Peter Schroder. 2004. Immersive Design of DNA Molecules with a Tangible Interface. In Proceedings of the Conference on Visualization '04 (VIS '04). IEEE Computer Society, Washington, DC, USA, 227-234. https://doi.org/10.1109/VISUAL.2004.47

[60] Bertrand Schneider, Megan Strait, Laurence Muller, Sarah Elfenbein, Orit Shaer, and Chia Shen. 2012. Phylo-Genie: Engaging Students in Collaborative 'Treethinking' Through Tabletop Techniques. In Proceedings of the SIGCHI Conference on Human Factors in Computing Systems (CHI '12). ACM, New York, NY, USA, 3071-3080. https://doi.org/10.1145/2207676.2208720

[61] Orit Shaer and Eva Hornecker. 2010. Tangible User Interfaces: Past, Present, and Future Directions. Foundations and Trends in Human-Computer Interaction 3, 1-2 (2010), 4-137. https://doi.org/10.1561/1100000026

[62] Orit Shaer, Ali Mazalek, Brygg Ullmer, and Miriam Konkel. 2013. From Big Data to Insights: Opportunities and Challenges for TEI in Genomics. In Proceedings of the 7th International Conference on Tangible, Embedded and Embodied Interaction (TEI '13). ACM, New York, NY, USA, 109-116. https://doi.org/10.1145/2460625.2460642

[63] O. Shaer, M. Strait, C. Valdes, H. Wang, T. Feng, M. Lintz, M. Ferreirae, C. Grote, K. Tempel, and S. Liu. 2012. The design, development, and deployment of a tabletop interface for collaborative exploration of genomic data. International fournal of Human-Computer Studies 70, 10 (2012), 746 - 764. https://doi.org/10.1016/j.ijhcs. 2012.05.003 Special issue on Developing, Evaluating and Deploying Multi-touch Systems.

[64] V. Siegel. 2016. The logic of journal embargoes: why we have to wait for scientific news. The Conversation. http://theconversation.com/ logic-of-journal-embargoes-why-we-have-to-wait-for-scientific-news-53677

[65] Aurélien Tabard, Juan-David Hincapié-Ramos, Morten Esbensen, and Jakob E. Bardram. 2011. The eLabBench: An Interactive Tabletop System for the Biology Laboratory. In Proceedings of the ACM International Conference on Interactive Tabletops and Surfaces (ITS '11). ACM, New York, NY, USA, 202-211. https: //doi.org/10.1145/2076354.2076391

[66] Brygg Ullmer, Zachary Dever, Rajesh Sankaran, Cornelius Toole Jr, Chase Freeman, Brooke Cassady, Cole Wiley, Mohamed Diabi, Alvin Wallace Jr, Michael DeLatin, et al. 2010. Cartouche: conventions for tangibles bridging diverse interactive systems. In Proceedings of the fourth international conference on Tangible, embedded, and embodied interaction. ACM, 93-100.

[67] B. Ullmer and H. Ishii. 2000. Emerging frameworks for tangible user interfaces. IBM Systems fournal 39, 3.4 (2000), 915-931. https://doi.org/10.1147/sj.393.0915

[68] Brygg Anders Ullmer and Hiroshi Ishii. 2002. Tangible interfaces for manipulating aggregates of digital information. Ph.D. Dissertation. Citeseer.

[69] Shaer-O. Mazalek A. Ullmer, B. and C. Hummels. 2019. Tangible and Embodied Interaction. (2019).

[70] John Underkoffler and Hiroshi Ishii. 1999. Urp: a luminous-tangible workbench for urban planning and design. In Proceedings of the SIGCHI conference on Human Factors in Computing Systems. ACM, 386-393.

[71] Consuelo Valdes, Diana Eastman, Casey Grote, Shantanu Thatte, Orit Shaer, Ali Mazalek, Brygg Ullmer, and Miriam K. Konkel. 2014. Exploring the Design Space of Gestural Interaction with Active Tokens Through User-defined Gestures. In Proceedings of the 32Nd Annual ACM Conference on Human Factors in Computing Systems (CHI '14). ACM, New York, NY, USA, 4107-4116. https://doi.org/10. $1145 / 2556288.2557373$

[72] Daniel Vogel and Patrick Baudisch. 2007. Shift: A Technique for Operating Penbased Interfaces Using Touch. In Proceedings of the SIGCHI Conference on Human Factors in Computing Systems (CHI '07). ACM, New York, NY, USA, 657-666. https://doi.org/10.1145/1240624.1240727

[73] Stephen Voida, Matthew Tobiasz, Julie Stromer, Petra Isenberg, and Sheelagh Carpendale. 2009. Getting Practical with Interactive Tabletop Displays: Designing for Dense Data, "Fat Fingers," Diverse Interactions, and Face-to-face Collaboration. In Proceedings of the ACM International Conference on Interactive Tabletops and Surfaces (ITS '09). ACM, New York, NY, USA, 109-116. https://doi.org/10.1145/ 1731903.1731926

[74] Daniel Wigdor, Hao Jiang, Clifton Forlines, Michelle Borkin, and Chia Shen. 2009. WeSpace: The Design Development and Deployment of a Walk-up and Share Multi-surface Visual Collaboration System. In Proceedings of the SIGCHI Conference on Human Factors in Computing Systems (CHI'09). ACM, New York, 
NY, USA, 1237-1246. https://doi.org/10.1145/1518701.1518886

[75] Kenneth G. Wilson. 1989. Grand challenges to computational science. Future Generation Computer Systems 5, 2 (1989), 171 - 189. https://doi.org/10.1016/ 0167-739X(89)90038-1 Grand Challenges to Computational Science.

[76] Ron Yeh, Chunyuan Liao, Scott Klemmer, François Guimbretière, Brian Lee, Boyko Kakaradov, Jeannie Stamberger, and Andreas Paepcke. 2006. ButterflyNet: A Mobile Capture and Access System for Field Biology Research. In Proceedings of the SIGCHI Conference on Human Factors in Computing Systems (CHI '06). ACM, New York, NY, USA, 571-580. https://doi.org/10.1145/1124772.1124859

[77] Jamie Zigelbaum, Michael S. Horn, Orit Shaer, and Robert J. K. Jacob. 2007. The Tangible Video Editor: Collaborative Video Editing with Active Tokens. In Proceedings of the 1st International Conference on Tangible and Embedded Interaction (TEI '07). ACM, New York, NY, USA, 43-46. https://doi.org/10.1145/ 1226969.1226978 